\title{
INFLUENCE DE LA TEMPÉRATURE SUR L'OVULATION DE L'OMBRE COMMUN THYMALLUS THYMALLUS
}

\author{
G. MAISSE (1) et H. CARMIE (2) \\ avec la collaboration technique de L. JONARD (2)
}

(1) INRA - Laboratoire de Physiologie des Poissons, 35042 RENNES Cedex, France

(2) CSP - Délégation Régionale Auvergne-Limousin, 84, avenue du Puy de Dóme, 63000 CLERMONT-FERRAND. France.

Recu le 12 Novembre 1986

Accepté le 11 Février 1987

Received 12 November, 1986

Accepted 11 February. 1987

\begin{abstract}
RÉSUMÉ
L'étude des courbes cumulées des ovulations chez l'Ombre commun montre qu une baisse de la température de l'eau dürant la période de la ponte ralentit ou inhibe l'ovulation. A partir de la relation entre la fréquence cumulée des ovulations et la somme des températures moyennes journalières calculée à partir du début de la période de ponte, nous avons montré l'existence d'un seuil minimal de température pour $\theta=5^{\circ} \mathrm{C}$. Un modèle prédictif est proposé pour aider le pisciculteur à décider de la fréquence des contrôles de maturité des femelles.
\end{abstract}

\section{INFLUENCE OF TEMPERATURE ON THE OVULATION OF THE GRAYLING (THYMALLUS THYMALLUS)}

\section{SUMMARY}

The study of the cumulative curves of the ovulations of the Grayling shows that the decrease of the water temperature during the spawning period reduces or inh ibits the ovulation process. Using the relationship between the cumulative frequency of ovulations and the sum of mean daily temperature from the beginning of the spawning period, we have shown a minimal thermal threshold for $\theta-5^{\circ} \mathrm{C}$. $A$ predictive model is given, to help the farmer to decide when he can check the females.

\section{INTRODUCTION}

La pisciculture de l'Ombre commun (Thymal/us thymal/us) est limitée à la production de jeunes sujets ( 0 -et 1 -) destinés aux déversements dans les rivières ou les lacs. Ce faible développement de l'élevage entraîne une domestication très peu poussée des individus rencontrés en pisciculture, qui sont alors très sensibles au stress. Ainsi, un trop grand nombre de manipulations peut perturber, voire inhiber totalement l'ovulation (STEIN ET WINTERSPERGER, 1980, CARMIE et al. 1985). CARMIE et al. (1985) ont montré qu'une chute brutale de la température provoque un ralentissement du rythme d'apparition des ovulations dans un lot de femelles d'élevage, et suggéré l'existence d'un seuil thermique minimal inhibiteur de $5^{\circ} \mathrm{C}$. Ils conseillent de tenir compte de ce seuil pour choisir les dates de contrôle de maturité des femelles. En France, la période d'ovulation de l'Ombre commun est printanière (PERSAT, 1984, CARMIE et al. 1985). La température de l'eau subissant de très grandes variations à cette saison, il nous a paru important de préciser l'existence de ce seuil thermique et, d'une manière plus générale, de modéliser le phénomène d'ovulation en fonction de la température. afin d'optimiser la fréquence de manipulation des géniteurs.

\section{MATÉRIEL ET MÉTHODES}

L'étude a porté sur les géniteurs (cohortes 1979 et 1982) de la pisciculture du C.S.P. à Augerolles (Puy-de-Dôme). Les nombres de femelles suivies ont été les suivants:

cohorte 1979: 88 en 1983, 83 en 1984, 40 en 1985, 17 en 1986

cohorte $1982: 142$ en 1985, 71 en 1986.

Chaque cohorte a été étudiée séparément sauf en 1986 où. en raison de son faible nombre et de l'absence de différence dans le déroulement des ovulations, la cohorte 1979 a été regroupée avec celle de 1982. 
Chaque année la fréquence cumulée des ovulations a été étudiée en fonction de la somme des températures moyennes journalières, exprimée en degrés-jours, et calculée à partir du "début" des ovulations (établi graphiquement).

Afin de définir un seuil thermique minimal (S.T.M.) en-dessous duquel les mécanismes de l'ovulation sont très ralentis, nous avons calculé la somme des températures moyennes journalières $(x)$ en ne tenant pas compte des températures inférieures ou égales à la valeur du seuil théorique. $\theta i \stackrel{x}{\mathbf{x}}$

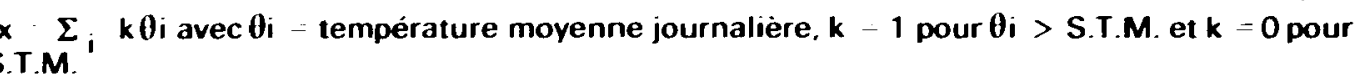

Nous avons ensuite recherché la valeur du seuil thermique minimal pour laquelle les courbes représentatives des fréquences cumulées des ovulations en fonction de la somme des degrés-jours sont les plus proches les unes des autres. Compte tenu des résultats de CARMIE et al. (1985) nous avons fait l'analyse pour les valeurs du seuil théorique $0^{\circ} \mathrm{C}, 4^{\circ} \mathrm{C}, 5^{\circ} \mathrm{C}$ et $6^{\circ} \mathrm{C}$.

En tenant compte du seuil thermique minimal le plus probable nous avons établi des modèles prédictifs de l'ovulation de l'Ombre commun en fonction des données thermiques par ajustement non linéaire au modèle logistique et à celui de Gompertz (modèles de croissance déterministes, cités par JOLIVET. 1982).

\section{RÉSULTATS}

La courbe représentative de la fréquence cumulée des ovulations en fonction de la date se présente sous 2 formes indépendantes de la cohorte: une forme en S (figures $1 B$ et $1 \mathrm{C}$ ) et une forme plus étirée (figures 1A et 1D). En 1983 les femelles subissent 2 chutes brutales de température; la
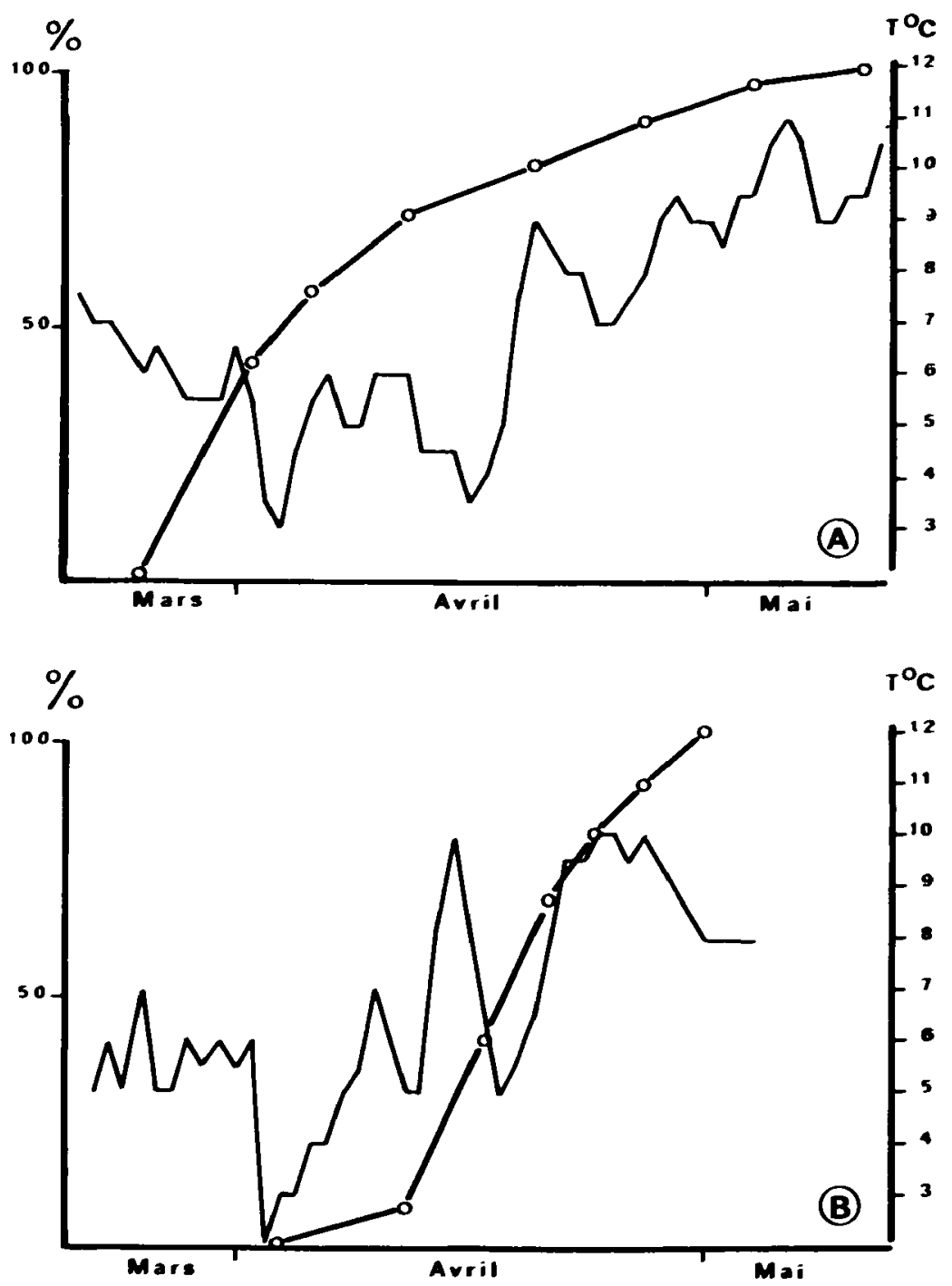

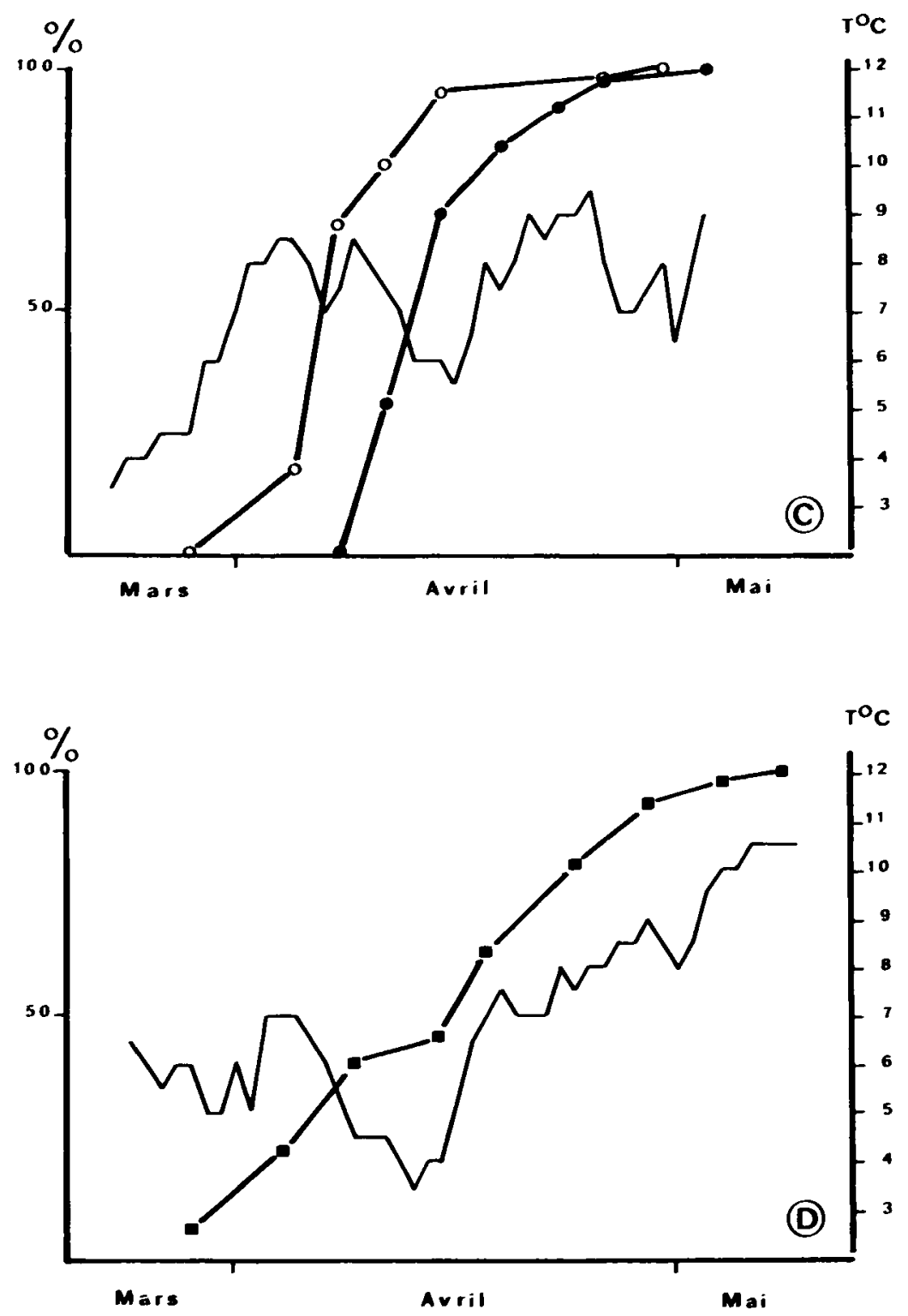

Figure 1 : - Fréquence cumulée des ovulations (\%) chez l'Ombre commun et température de l'eau $\left(T^{\circ} \mathrm{C}\right)$ suivant la date.

- Année de ponte : $A=1983 ; B=1984 ; C=1985 ; D=1986$

- Cohorte de géniteurs: $0-0: 1979 ; 0: 1982 ; \square-1979+1982$

Figure 1 : - Cumulative frequency of ovulations $(\%)$ in the Grayling and water temperature $\left(T^{\circ} \mathrm{C}\right)$ according to the date.

- Spawning year : $A=1983 ; B=1984 ; C=1985 ; D=1986$

- Group of spawners : $\bigcirc-0$ 1979; $\longrightarrow 1982 ; \square 1979+1982$

première, après que $\mathbf{4 5} \%$ des femelles aient ovulé, coïncide avec une diminution de la pente de la courbe. En 1986, une chute semblable apparaît après que $40 \%$ des femelles aient ovulé ; elle est contemporaine d'un palier de la courbe d'ovulation. En 1984 et 1985 la forme des courbes d'ovulation est en $S$ et il n'y a pas eu de chute de la température en dessous de $5^{\circ} \mathrm{C}$, après le début des ovulations. En 1984 la ponte n'avait pas encore commencé quand le refroidissement a eu lieu début Avril.

La figure 2 montre que c'est pour un seuil thermique minimal théorique de $5^{\circ} \mathrm{C}$ que les courbes représentatives des fréquences cumulées des ovulations en fonction de la somme des températures moyennes journalières sont les plus proches les unes des autres (figure $2 \mathrm{C}$ ). 
Bull. Fr. Pêche Piscic. (1987) $305: 54-60-57-$
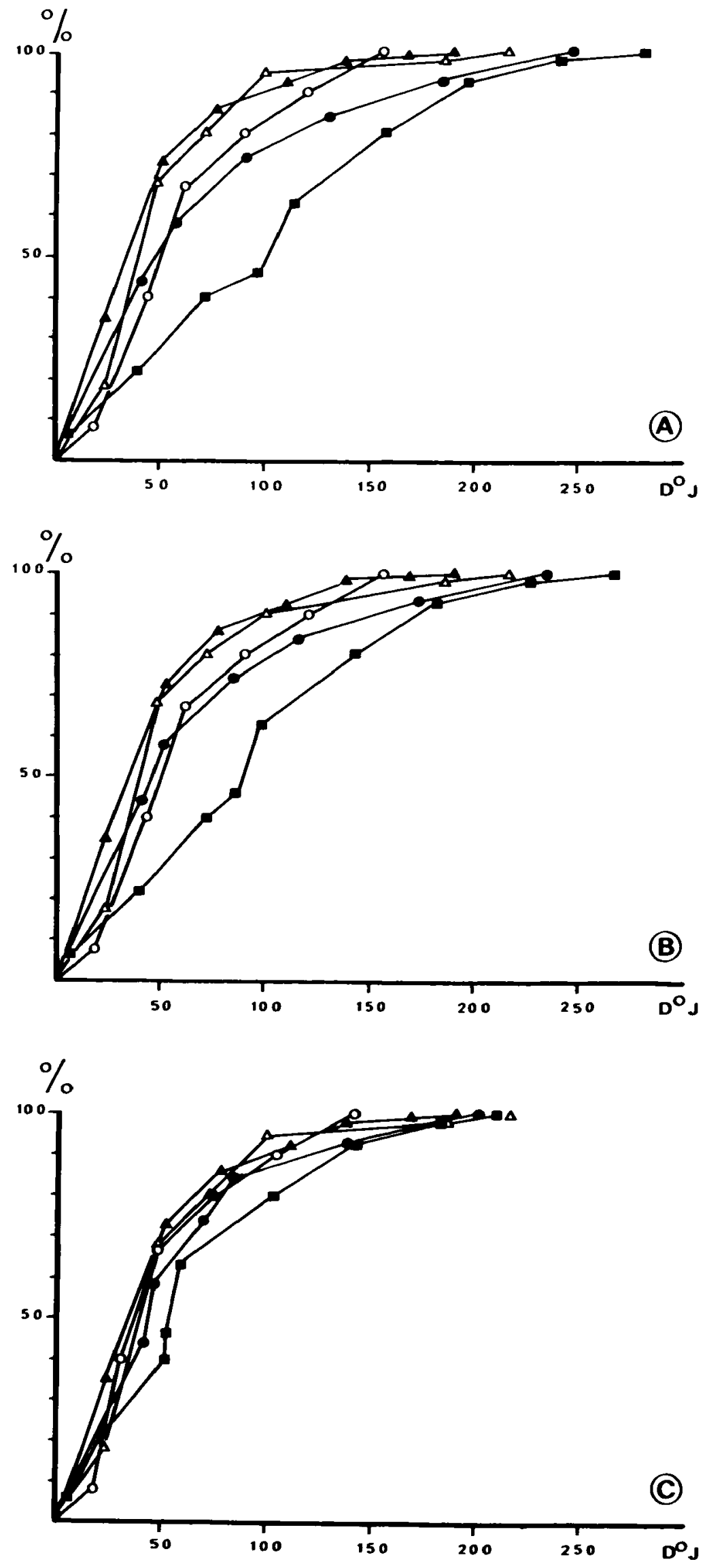


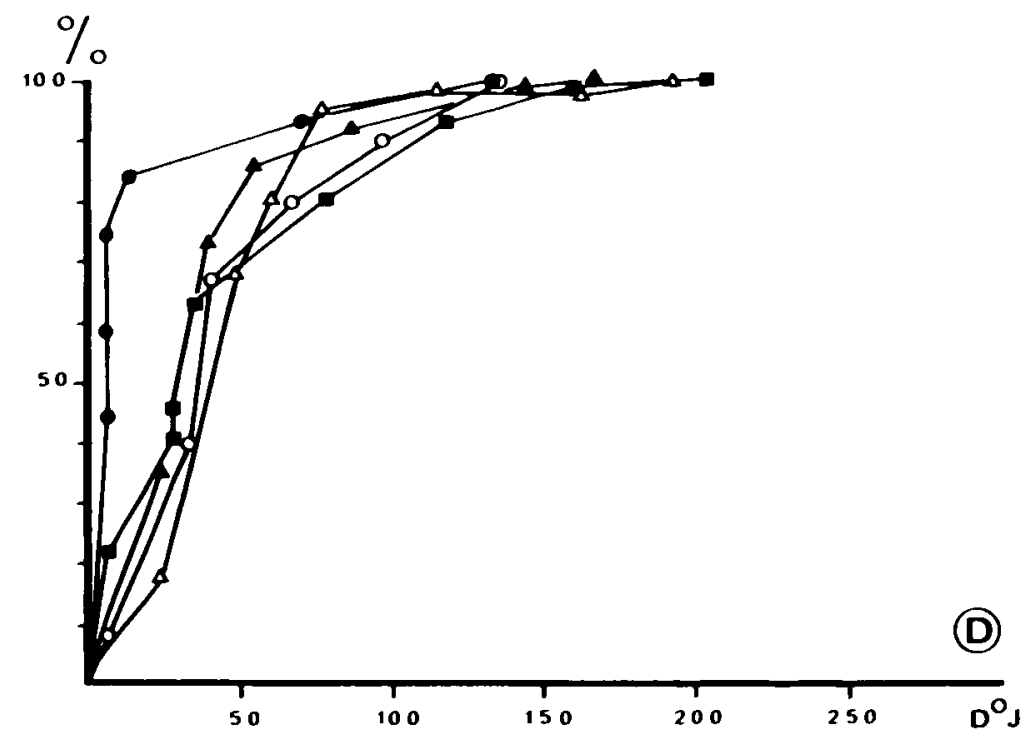

Figure 2 : - Fréquence cumulée des ovulations $(\%)$ chez l'Ombre commun en fonction de la somme des températures moyennes journalières $\left(D^{\circ} \mathrm{J}\right): x=\Sigma ; k \theta_{i}$ avec $\theta_{1}=$ température moyenne journalière

$$
\begin{aligned}
& k=1 \text { pour } \theta>\text { S.T.M. } \\
& k=0 \text { pour } \theta \leq \text { S.T.M. }
\end{aligned}
$$

Seuil thermique minimal (S.T.M.): $A=0^{\circ} \mathrm{C} ; \mathrm{B}-4^{\circ} \mathrm{C} ; \mathrm{C}=5^{\circ} \mathrm{C} ; \mathrm{D}=6^{\circ} \mathrm{C}$.

Géniteurs/Année : ○- $079 / 1983 ; \bullet \longrightarrow 79 / 1984 ; \Delta-\Delta 79 / 1985$; $\triangle \longrightarrow$-A2/1985; — $(79-82) / 1986$.

Figure 2 : - Cumulative frequency of ovulations (\%) in the Grayling according to the sum of mean daily temperatures $\left(D^{\circ} \mathrm{J}\right): x=\Sigma_{i} k \theta_{i}$ with $\theta_{\mathrm{i}}=$ mean daily temperatures

$$
k=1 \text { when } \theta>\text { S.T.M. }
$$$$
\text { k }=\mathbf{O} \text { when } \theta \leqslant \text { S.T.M. }
$$

minimal thermal level (S.T.M.): A: $0^{\circ} \mathrm{C}: \mathrm{B}: 4^{\circ} \mathrm{C}: \mathrm{C}: 5^{\circ} \mathrm{C}: \mathrm{D}: 6^{\circ} \mathrm{C}$.

Spawners/year : $0 \longrightarrow 079 / 1983 ; \bullet \longrightarrow 79 / 1984: \Delta \longrightarrow \Delta 79 / 1985 ; \triangle \longrightarrow \Delta 82 / 1985$ : $\stackrel{\sim}{\longrightarrow}(79-82) / 1986$.

Pour ce seuil de $5^{\circ} \mathrm{C}$, le tableau 1 donne les équations de la courbe représentative de l'ensemble des points suivants les 2 modèles choisis.

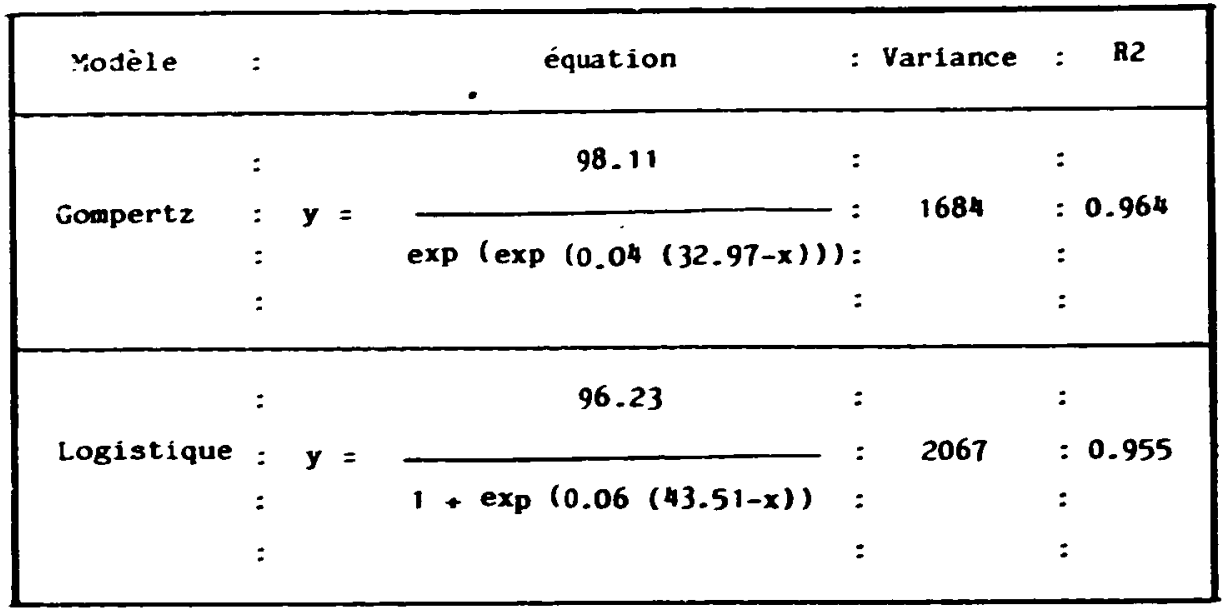

Tableau 1 : Relation entre la fréquence cumulée des ovulations ( $y$ ) et la somme des degrés jours $(x)$ depuis le début de la période de ponte. Remarque: pour chacun des modéles il n'y a pas de corrélation entre les paramètres calculés. 


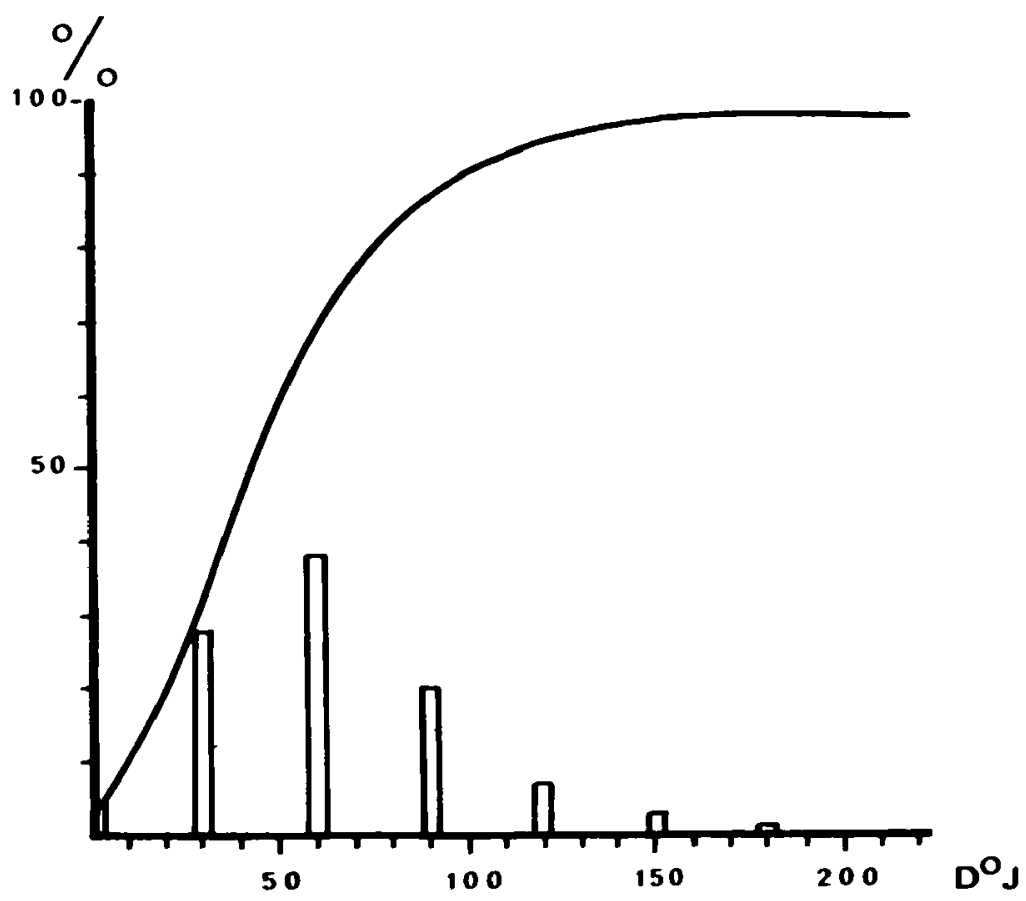

Figure 3 : - Courbe représentative du modèle prédictif des ovulations en fonction de la température chez l'Ombre commun.

$$
y=\frac{98.11}{\exp (\exp (0.04(32.97-x)))}
$$

avec:

$y=$ fréquence cumulée des ovulations

$x=$ somme des degrés-jours

$\hat{x}=\Sigma_{i} k \theta_{i}, \theta i=$ température moyenne journalière

$$
\begin{aligned}
& k=1 \text { quand } \theta i>5^{\circ} \mathrm{C} \\
& k=\theta \text { quand } \theta i \leqslant 5^{\circ} \mathrm{C}
\end{aligned}
$$

L'histogramme de fréquence des ovulations est donné à tître indicatif pour des intervalles de $\mathbf{3 0}$ degrés-jours.

Figure 3 : - Représentative curve of the predictive model of the relationship between the ovulations and the temperatures in the Grayling.

\subsection{1}

$$
y=\frac{\exp (\exp (0.04(32.97-x))}{9.11}
$$

with:

$y$ : cumulative frequency of ovulations

$x$ : Sum of degree-days

$x: \Sigma_{i} k \theta_{i}, \theta_{i}=$ average daily temperature

$$
\begin{aligned}
& k=1 \text { when } \theta i>5^{\circ} \mathrm{C} \\
& k=\theta \text { when } \theta i \leqslant 5^{\circ} \mathrm{C}
\end{aligned}
$$

histogramm gives the predicted frequencies at each interval of 30 degree-days.

\section{DISCUSSION}

La comparaison des profils des courbes de la figure 1 permettent d'envisager le rôle inhibiteur joué par les refroidissements brutaux qu'ont eu à subir les femelles en 1983 et 1986, alors que près de la moitié avait pondu. Il est à noter que la forme de la courbe d'ovulation dépend non seulement de l'existence de la chute de température. mais aussi de sa durée. En 1986. un véritable palier apparaît au moment d'un refroidissement d'une durée de 8 jours, alors qu'en 1983 seul un ralentissement est noté pour une chute thermique de 3 jours.

Lutilisation de la somme des températures moyennes journalières permettant une comparaison entre les années, son mode de calcul ne tenant pas compte des températures inférieures au seuil confirme le rôle inhibiteur de lovulation joué par les températures inférieures ou égales à $5^{\circ} \mathrm{C}$. 
Ces observations vont dans le sens des conclusions de BILLARD et al. (1981) qui considèrent que les espèces pisciaires de la zone tempérée subissent l'influence des fluctuations thermiques particulièrement au niveau de la spermiation, de la maturation ovocytaire, de l'ovulation et de l'oviposition.

La sensibilité de l'Ombre commun à une chute de température est mise d'autant plus facilement en évidence que sa fourchette de température optimale de fraie est une des plus étroites parmi celle des poissons de la zone tempérée: 6-10 ${ }^{\circ} \mathrm{C}$, pour l'Ombre commun, 4-19 ${ }^{\circ} \mathrm{C}$ pour la Truite arc-en-ciel (Salmo gairdneri), 4-17 ${ }^{\circ} \mathrm{C}$. pour le Brochet (Esox lucius), 8-24 ${ }^{\circ} \mathrm{C}$ pour la Brème (Abramis brama), (voir la revue bibliographique d'ELLIOT, 1981).

Par ailleurs il faut préciser que notre analyse. à partir des températures moyennes journalières. ne rend pas compte de l'influence éventuelle de fluctuations au cours de la journée, auxquelles l'Ombre commun est sensible tant pour la migration de fraie (GUSTAFSON. 1949), que pour l'oviposition (FABRICIUS et GUSTAFSON. 1955).

Enfin, il faut noter la coincidence entre la valeur du seuil que nous avons mise en évidence et celle trouvée par JUNGWIRTH et WINKLER (1984) pour l'incubation : le pourcentage de mortalité est de $10 \%$ pour une température d'incubation de $5.5^{\circ} \mathrm{C}$ et de $90 \%$ pour $4^{\circ} \mathrm{C}$.

\section{CONCLUSION}

Le modèle de Gompertz pour lequel la variance est la plus faible a été choisi de préférence au modèle logistique. pour réaliser la courbe de prédiction des ovulations en fonction de la somme des températures moyennes journalières depuis la date de la première ovulation (figure 3). CARMIE et al. (1985) ont proposé de fixer cette date pour la pisciculture d'Augerolles à environ 130 degrés-jours après le 1 er mars. Si, d'une manière globale les résultats obtenus en 1985 et 1986 (125 degrés-jours) confirment les premières observations, il faut remarquer que pour la cohorte 82 la première ovulation est apparue à 165 degrés-jours en 1985. Il est possible que le décalage observé soit dù au fait que la cohorte 82 était dans son premier cycle sexuel en 1985 . Compte tenu du manque de connaissances fondamentales sur le cycle reproducteur de l’Ombre commun et en particulier sur le rôle joué par les facteurs externes (photopériode, cycles thermiques) il nous est difficile de préciser le déterminisme de la date de première ovulation. Enfin, nous avons présenté dans cette figure 3, sous forme d'histogramme. le pourcentage de femelles ovulées prévisible à des intervalles de 30 degrés-jours (température $>5^{\circ} \mathrm{C}$ ), compatibles avec une bonne qualité des ovules (CARMIE et al. 1985).

\section{REMERCIEMENTS}

Messieurs LOMBARDY et MESTAS, employés à la pisciculture d'Augerolles ont assuré l'entretien des géniteurs et participé à la collecte des données.

\section{BIBLOGRAPHIE}

BILLARD R.. BRY C.. GILLET C., 1981. Stress, environment and reproduction in teleost fish. In PICKERING A.D., Stress and fish, 185-208, Academic Press Inc. (London) Ltd.

CARMIE H., MORELET B., MAISSE G., JONARD L., CUINAT R., 1985. Observations sur la reproduction artificielle de l'Ombre commun (Thymallus thymallus). Bull. Fr. Pêche Piscic., 296. 2-16.

ELUOT J.M., 1981. Some aspects of thermal stress on freshwater teleosts. In PICKERING A.D., Stress and fish, 211-245. Academic Press Inc. (London) Ltd.

FABRICIUS E., GUSTAFSON K.J., 1955. Observations on the spawning behaviour of the Grayling, Thymallus thymallus (L.). Ann. Rep. Inst. Freshwater Res. Drottningholm, 36. 75-103.

GUSTAFSON K.J., 1949. Movements and growth of Grayling. Ann. Rep. Inst. Freshwater Res. Drottningholm, 29, 35-44.

JOLIVET E., 1982. Introduction aux modèles mathématiques en biologie. INRA, Actualités scientifiques et agronomiques, 11, Masson, Paris, $151 \mathrm{p}$.

JUNGWIRTH M., WINKLER H., 1984. The temperature dependance of embryonic development of Grayling (Thymallus thymallus), Danube salmon (Hucho hucho), Arctic char (Salvelinus alpinus) and Brown trout (Salmo trutta fario). Aquaculture, 38, 315-327.

PERSAT H., 1984. Etude de la reproduction de l'Ombre commun. Rapport technique. Départ. Biol. Anim. Ecol. Université Lyon I, 22 p.

STEIN H. WINTERSPERGER R., 1980. The artificial insemination of the Grayling (Thyma/lus thymallus L.). 9th International Congress on Animal Reproduction Madrid (1980). vol. 11 : 311-315. 